\title{
Landmark-Based Navigation in Cognitive Systems
}

\author{
Jakub Krukar $^{1} \cdot$ Angela Schwering $^{1} \cdot$ Vanessa Joy Anacta $^{1}$
}

Published online: 10 March 2017

(C) Springer-Verlag Berlin Heidelberg 2017

\begin{abstract}
The current special issue of KI Journal brings together a collection of articles related to landmark-based navigation. This includes the problems of identifying suitable landmark candidates in a scalable manner, selecting landmarks which are relevant to the navigating agent, communicating their presence on maps, and integrating them into location-based services.
\end{abstract}

The decades-long prominence of landmark studies demonstrates how an intuitively understood concept can inspire research stretching across the boundaries of formally defined academic disciplines. Research on landmarks has been continuing for a period long enough, ${ }^{1}$ to allow some of the involved disciplines (e.g. psychology) to go through multiple paradigm shifts and new academic fields (e.g. AI) to develop. Recently, landmarks have begun to appear in commonly accessible technological applications such as incar navigation systems. While the problem of selecting and depicting landmarks for centuries remained primarily the domain of a cartographers' design choice, in the recent decades the need for formalising and generalising what landmarks are and how to communicate them has become evident.

Guest editors are supported by the German Research Foundation (DFG) with Project Number SCHW1371/15-1 and the ERC StRG Grant Agreement No. 637645.

Jakub Krukar

krukar@uni-muenster.de

1 Institute for Geoinformatics, University of Münster, Heisenbergstr. 2, 48149 Münster, Germany
A fact testifying to how long and diverse these investigations are, seems to be the number of labels and classifications that landmarks have been subjected to. They have been defined with terms such as global versus local landmarks [11, 15], structurally salient versus perceptually salient landmarks [6, 9], or point-based versus regional ones $[13,14]$. Perhaps the variety of existing definitions is signifying the scope of interests with which researchers of distinct academic backgrounds approached the issue. While some fields are interested in the nature of mental representations of landmarks and their relation to other aspects of spatial knowledge [10], others ask the question of what makes a spatial object stand out as a landmark [2] (and does it always need to be an 'object'; [7]), how landmarks can be used in navigation of artificial systems [3], or communicated on various maps [8]. The seminal book on the issue [12] (see Ishikawa's review in the current issue) is formally organised around four distinct, but interacting, 'aspects' of landmarks: the cognitive, the conceptual, the computational, and the communicational. Each of them provided ground for studies across a diverse set of disciplines. It is therefore not very surprising that 'landmarks' may mean different things, depending on the context in which they are being discussed. This concern puts its mark on the content of the current special issue of the German Journal of Artificial Intelligence in two Discussion articles.

Despite not lending itself easily to formal definitions, landmarks certainly are an important, intuitively understood lay notion. Their most recent popular non-academic application is visible in the augmented reality game "Pokemon GO". In this special issue, its impact is reflected in

\footnotetext{
${ }^{1}$ Montello in this issue points to Trowbridge's 1913 paper as one of
} the first mentions. 
the fact that two authors have independently decided to add references to the game to their revised drafts throughout a 3 months-long reviewing process, while the game was gaining its peak popularity. It is worth noting that this prominent recent use of landmarks in a popular geographic information system has been crowd-sourced (via an earlier game "Ingress"), as opposed to being modeled computationally from a set of predefined parameters. This recognition of approaches involving direct or indirect human judgment is also reflected in the current issue of the Journal.

Making any landmark selection system dependent on human judgment comes with an obvious caveat. People widely differ in what they consider a useful landmark and groups of individuals may distinguish different sets of objects as potential landmarks in distinct types of environments $[1,4]$. Temporal context, such as changing seasons [5] and the day/night cycle [16], play a further role in this process. Consequently, it is quite likely that no generalisation will ever be able to satisfy all potential use-cases, for all potential users. This is one point where dissimilarities in research interests of distinct disciplines are so clearly visible. While technology developers would be often satisfied with identifying 'safe' landmark candidates-easy to agree on for all of us, other fields are interested in generalizable explanations of where those individual disparities come from. As a result, there is a gap between what the technologically oriented approach might sometimes call cognitively '-plausible', '-inspired', or '-appropriate' and our actual understanding of the relevant cognitive representations and processes.

Another matter deserving attention is the seemingly understudied concept of indoor landmarks, at least when compared to its outdoor counterpart (see Krisp as well as Müller et al. in the current volume). In the wayfinding context this is the topic of this issue's interview with an architect, Ruth Conroy Dalton, and a cognitive psychologist Christoph Hölscher. The interviewees point to distinct processes and environmental differences involved in the indoor navigation compared to its outdoor counterpart. This raises the question of how many findings established in the outdoor landmark studies are directly applicable indoors.

The current special issue of the German Journal on Artificial Intelligence contains, among project descriptions, dissertation abstracts, conference reports and news, four technical contributions, two discussion articles, the abovementioned interview and a book review.

Richter concentrates on the issue of scalability for selecting landmark candidates. He envisions a system that would use a combination of generic top-down selection based on the object's type with a feedback mechanism allowing users to spontaneously contribute refinements of the initial classification.
Müller and colleagues validate a landmark-based indoor navigation system using an interesting approach of comparing the properties of routes generated by an algorithm to those resulting from a spontaneous navigational choice of their participants. The authors use landmarks as an interface for providing indoor routing instructions more suited to the human navigators than traditional approaches optimised around the shortest or quickest path. The proposed weighting system associated with the representation of the indoor environment provides a platform for validating possible modifications to the algorithm.

Kim et al. demonstrate a method for deriving landmarks from web-harvested place descriptions, utilising large corpora of potentially relevant landmark information generated by humans for a variety of original purposes. They particularly focus on Wikipedia, as well as travel and business resources, demonstrating the flexibility of their automated approach across different semantic contexts.

$\mathrm{Li}$ and Zhao concentrate on the issue of visualising landmark information. They evaluate alternative means of presenting off-screen landmarks on mobile devices in a way that would be intuitively understood by the users. By doing so, they demonstrate the potential of distant landmarks to organise even this spatial information, which is not directly accessible on the currently visible section of the map.

In his Discussion essay, Montello questions the consistency with which the term 'landmark' is referred to in research. He notes how the single term is used to describe a multitude of distinct concepts. This may lead to exaggerated interpretations of research findings that are never meant to jointly refer to all of the terms falling under the broad 'landmark umbrella'. The author also points out how landmarks are believed to define locations and how this assumption contributes to making landmarks an exaggerated research concept.

In another Discussion article, Krisp reviews the grounds for including landmarks in Location-Based Services. He reminds us about older navigation support systems and envisions possible future developments that have (and can) function well without landmarks. He suggests that, despite not being needed from the functional perspective, landmarks play an important role for multiple other reasons, for instance serving as meeting points or enhancing potential for personalisation.

The topics of these contributions seem to centre around what currently are the focal issues of landmarkbased studies:

- the need for a more explicit definition of landmarks and 'landmarkness';

- convergence of approaches for identifying and selecting landmarks between well-formalised, top-down 
modeling and non-scalable, human-dependent, caseby-case selection;

- using landmarks to support human navigation in a more user-friendly manner, both in the outdoor and in the indoor context.

All technical contributions were reviewed by two or three external peers. Discussion articles were reviewed by two peers with the focus on the clarity of the argument but not on the opinion of the author. We would sincerely like to thank all authors, reviewers and our liaising editor Sabine Timpf for joining the effort in making this special issue possible. Finally, one message learnt from preparing this work which we consider worth sharing with all authors reporting landmark-related studies is the following: being explicit about the exact understanding of the term 'landmark' in one's study makes it possible to evaluate the report in a wider context, beyond the single discipline or the single use-case in question. We hope the reader finds this special issue worthwhile and representative of the current state-of-affairs in the field.

\section{Content}

\subsection{Technical Contributions}

- Manuel Müller, Christina Ohm, Florin Schwappach, Bernd Ludwig: The Path of Least Resistance: Calculating Preference Adapted Routes for Pedestrian Navigation

- Kai-Florian Richter: Identifying Landmark Candidates Beyond Toy Examples: A Critical Discussion and Some Way Forward

- Rui Li, Jiayan Zhao: Resizing Off-screen Landmarks on Mobile Devices: Levels of Measurement and the Perception of Distance

- Junchul Kim, Maria Vasardani, Stephan Winter: Landmark Extraction from Web-harvested Place Descriptions.

\subsection{Dissertations and Project Abstracts}

- Florian Röser: A Cognitive Observer-based Landmark-preference Model

- Markus Kattenbeck: Empirically Measuring Salience of Objects for Use in Pedestrian Navigation

- Anne-Kathrin Bestgen, Dennis Edler, Lars Kuchinke, Frank Dickmann: Analyzing the Effects of VGI-based Landmarks on Spatial Memory and Navigation Performance.

\subsection{Interviews}

- Indoor Wayfinding: Interview with Christoph Hölscher and Ruth Conroy Dalton.

\subsection{Discussions}

- Daniel R. Montello: Landmarks are Exaggerated

- Jukka M. Krisp: Landmarks for Location-based Services (LBS) in Particular Navigation and Wayfinding.

\subsection{Book Reviews}

- Toru Ishikawa: Book review of "Landmarks: GIScience for Intelligent Services".

\section{Service}

\subsection{Major Projects and Organisations}

- ICA Commission on Location Based Services: http:// lbs.icaci.org

- American Association of Geographers: http://www. aag.org

- Association of Geographic Information Laboratories in Europe (AGILE): https://agile-online.org

- Spatial Intelligence and Learning Center (SILC): http://spatiallearning.org.

\subsection{Journals}

- Journal of Location Based Services: http://www.tandfonline.com/toc/tlbs20/current

- Computers, Environment and Urban Systems: https:// www.journals.elsevier.com/computers-environmentand-urban-systems

- International Journal of Geographical Information Science: http://www.tandfonline.com/toc/tgis20/current

- Spatial Cognition \& Computation: http://www.tandfonline.com/toc/hscc20/current

- Cognitive Processing: http://link.springer.com/journal/10339

- Cartographica: The International Journal for Geographic Information and Geovisualization: http://www. utpjournals.press/loi/cart

- Cartography and Geographic Information Science: http://www.tandfonline.com/toc/tcag20/current

- Journal of Spatial Information Science: http://www. josis.org

- Geoinformatica: http://link.springer.com/journal/10707 
- Transactions in GIS: http://onlinelibrary.wiley.com/ journal/10.1111/(ISSN)1467-9671

- Journal of Environmental Psychology: https://www. journals.elsevier.com/journal-of-environmental-psychology.

\subsection{Conferences}

- Conference on Spatial Information Theory (COSIT): http://www.cosit.info

- GIScience: http://www.giscience2016.org

- Spatial Cognition Conference http://bscc.spatial-cognition.de/node/6

- AGILE Conference: https://agile-online.org

- ACM SIGSPATIAL: http://www.sigspatial.org.

\subsection{Open Software and Datasets}

- OpenStreetMap: https://www.openstreetmap.org

- Wikimapia: http://wikimapia.org

- OpenLS (OGC): http://www.opengeospatial.org/standards/ols.

\subsection{Commercial Hardware and Software}

- HERE Maps: https://here.com

- Foursquare: https://foursquare.com.

\section{References}

1. Brosset D, Claramunt C, Saux E (2008) Wayfinding in natural and urban environments: a comparative study. Cartographica 43(1):21-30

2. Caduff D, Timpf S (2008) On the assessment of landmark salience for human navigation. Cognit Process 9(4):249-267. doi:10.1007/s10339-007-0199-2

3. Dudek G, Jenkin M (2010) Computational principles of mobile robotics. Cambridge University Press, Cambridge
4. Egorova E, Tenbrink T, Purves RS (2015) Where snow is a landmark: route direction elements in alpine contexts. In: Spatial information theory. Springer, New York, pp 175-195. doi:10.1007/978-3-319-23374-1_9

5. Kettunen P, Irvankoski K, Krause CM, Sarjakoski LT (2013) Landmarks in nature to support wayfinding: the effects of seasons and experimental methods. Cognit Process 14(3):245-253

6. Klippel A, Winter S (2005) Structural salience of landmarks for route directions. In: Cohn AG, Mark DM (eds) Proceedings of the spatial information theory: international conference, COSIT 2005, COSIT'05, Ellicottville, 14-18 September 2005. Springer, Berlin, pp 347-362. doi:10.1007/11556114_22

7. Lynch K (1960) The image of the city. MIT Press, London

8. MacEachren AM (1986) A linear view of the world: strip maps as a unique form of cartographic representation. Am Cartogr 13(1):7-26

9. Miller J, Carlson L (2011) Selecting landmarks in novel environments. Psychon Bull Rev 18(1):184-191. doi:10.3758/ s13423-010-0038-9

10. Montello DR (1998) A new framework for understanding the acquisition of spatial knowledge in large-scale environments. In: Egenhofer M (ed) Spatial and temporal reasoning in geographic information systems. Oxford University Press, New York, pp 143-154

11. Raubal M, Winter S (2002) Enriching wayfinding instructions with local landmarks. Proceedings of the second international conference on geographic information science 2478:243-259. doi:10.1007/3-540-45799-2_17

12. Richter KF, Winter S (2014) Landmarks-GIScience for intelligent services. Springer, Cham. doi:10.1007/978-3-319-05732-3

13. Schmid F, Richter KF, Peters D (2010) Route aware maps: multigranular wayfinding assistance. Spat Cognit Comput 10(23):184-206. doi:10.1080/13875861003592748

14. Schwering A, Li R, Anacta VJA (2013) Orientation information in different forms of route instructions. In: Short paper proceedings of the 16th AGILE conference on geographic information science, Leuven

15. Steck S, Mallot $\mathrm{H}$ (2000) The role of global and local landmarks in virtual environment navigation. Presence Teleoper Virtual Environ 9(1):69-83

16. Winter S, Raubal M, Nothegger C (2005) Focalizing measures of salience for wayfinding. In: Map-based mobile services. Springer, New York, pp 125-139 